\title{
4D Flow MRI quantification of blood flow patterns, turbulence and pressure drop in normal and stenotic prosthetic heart valves
}

Hojin Ha, J ohn-Peder Escobar Kvitting, Petter Dyverfeldt and Tino Ebbers

The self-archived postprint version of this journal article is available at Linköping University Institutional Repository (DiVA):

http:/ / urn.kb.se/ resolve?urn=urn:nbn:se:liu:diva-153497

N.B.: When citing this work, cite the original publication.

Ha, H., Escobar Kvitting, J., Dyverfeldt, P., Ebbers, T., (2019), 4D Flow MRI quantification of blood flow patterns, turbulence and pressure drop in normal and stenotic prosthetic heart valves, Magnetic Resonance Imaging, 55, 118-127. https:// doi.org/ 10.1016/j.mri.2018.09.024

Original publication available at:

https:/ / doi.org/ 10.1016/j.mri.2018.09.024

Copyright: Elsevier

http:// www.elsevier.com/

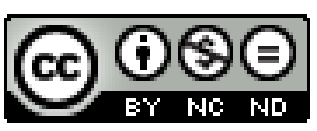




\section{D Flow MRI Quantification of Blood Flow Patterns, Turbulence and Pressure Drop in}

Normal and Stenotic Prosthetic Heart Valves

Hojin $\mathrm{Ha}^{1,2,3^{*}}$, John-Peder Escobar Kvitting ${ }^{2,3,4}$, Petter Dyverfeldt ${ }^{2,3}$, Tino Ebbers ${ }^{2,3}$

${ }^{1}$ Department of Mechanical and Biomedical Engineering, Kangwon National University, Chuncheon, Republic of Korea.

${ }^{2}$ Division of Cardiovascular Medicine, Department of Medical and Health Sciences, Linköping University, Linköping, Sweden.

${ }^{3}$ Center for Medical Image Science and Visualization (CMIV), Linköping University, Linköping, Sweden.

${ }^{4}$ Department of Cardiothoracic Surgery, Oslo University Hospital, Rikshospitalet, Oslo, Norway.

Corresponding Author: Hojin $\mathrm{Ha}^{*}$

Department of Mechanical and Biomedical Engineering,

Kangwon National University,

Chuncheon, Republic of Korea.

Phone: +82-1092871005

Fax: +82-33-259-5548

E-mail: hojinha@kangwon.ac.kr 


\section{Abstract}

Purpose - To assess valvular flow characteristics and pressure drop in a variety of normal and stenotic prosthetic heart valves (PHVs) using 4D Flow MRI.

Materials and methods - In-vitro flow phantoms with four different PHVs were studied: Medtronic-Hall tilting disc, St. Jude Medical standard bileaflet (STJM), Medtronic CoreValve Evolut $\mathrm{R}$ and Edwards SAPIEN 3. The valvular flow characteristics were investigated in normal and stenotic PHVs by using 4D Flow MRI.

Results - The results showed that each valve provided a different amount of signal loss in the 4D Flow MRI. The defect size of the signal loss from each valve was $37.5 \mathrm{~mm}, 39.0 \mathrm{~mm}$, $37.5 \mathrm{~mm}$ and $51.0 \mathrm{~mm}$ for the Tilting disk, STJM, SAPIEN 3 and CoreValve, respectively. The 4D Flow MRI-based estimation of the elevation of the pressure drop through the stenotic PHV using both Bernoulli-based and turbulence-based methods correlated well with the true values for the Tilting disc, STJM and SAPIEN 3 valve. However, the obstructive hemodynamics in the stenotic CoreValve was not clearly identified due to the large signal void from the long struts, resulting in a severe underestimation of the pressure drop using 4D Flow MRI.

Conclusion - The Tilting disc, STJM and SAPIEN 3 valves provided reasonable estimates of peak velocity, turbulence production and the corresponding pressure drop. In contrast, the large strut of the CoreValve and corresponding signal void prevented accurate measurements of the velocity and turbulence production; therefore, 4D Flow MRI prediction of the pressure drop through the CoreValve was not feasible.

Key words: turbulence; Reynolds stress; pressure drop; magnetic resonance imaging; phase contrast MRI; 4D PC-MRI; 4D Flow MRI 


\section{Abbreviations}

PHV, Prosthetic Heart Valves; TTE, Transthoracic Echocardiography; CMR, Cardiovascular Magnetic Resonance; TAVI, Transcatheter Aortic Valve Implantation; VENC, Velocity Encoding ranges; TE, Echo time; TKE, Turbulence Kinetic Energy; IVVV, Intravoxel Velocity Variance. 


\section{Introduction}

The replacement of diseased heart valves with prosthetic heart valves (PHVs) has saved numerous lives since the first implant in the early 1960s. Approximately 90,000 patients receive a PHV each year in the United States and 280,000 worldwide [1, 2]. The continuous improvement in surgical and interventional procedures will lead to an increasing number of patients living with a PHV in the future.

Replacement of diseased heart valves is a life-saving procedure; however, PHVs are associated with several complications, such as the risk of thromboembolism, hemolysis, bleeding, prosthesis-patient mismatch, structural valve deterioration and endocarditis [3]. Several of these complications can be prevented or treated with careful monitoring of the PHV and appropriate management. Early detection and accurate assessment of these complications are pertinent for the long-term surveillance of patients with a PHV $[3,4]$.

Transthoracic echocardiography (TTE) has been widely used to evaluate the function and possible dysfunction of PHVs [1]. A significant increase in transvalvular flow velocity and corresponding pressure drop has been suggestive of PHV dysfunction. However, TTE can be observer-dependent and mean or peak velocity of the flow provides only limited information about the valvular blood flow, and is occasionally misleading as to the severity of the stenosis [5-8]. Therefore, assessment of the hemodynamics using cardiovascular magnetic resonance (CMR) has been proposed as a complement to the conventional TTE evaluation as it is independent of the anatomy of the patient and less observer-dependent [2, 9].

Time-resolved, three-directional and three-dimensional phase-contrast magnetic resonance imaging (which is widely known as 4D PC-MRI or 4D Flow MRI) has been demonstrated to provide non-invasive and multi-dimensional hemodynamic analysis [10-12]. 4D Flow MRI not only provides a three-dimensional velocity field of the blood flow, but also 
reveals the extent and degree of turbulence downstream of the PHV, which is suggestive of abnormalities in the blood flow [9]. Assessment of the turbulence level of the blood flow using 4D Flow has been proposed to evaluate flow-induced blood damage to blood constituencies and also the pressure drop observed in stenotic blood flow [13, 14].

Hemodynamics in various types of PHVs, however, have rarely been investigated using 4D Flow MRI $[9,15,16]$ and data on PHVs used in transfemoral or transapical aortic valve implantation (TAVI) are limited [17]. Therefore, normal and abnormal levels of the hemodynamics in each PHV and the feasibility of the 4D Flow MRI for detecting the abnormality of the hemodynamics in the PHV remain to be elucidated.

The purpose of this study was: (1) to assess the valvular flow characteristics in various PHVs using 4D Flow MRI and (2) to investigate the feasibility of 4D Flow MRI measurement for detecting abnormality in PHVs and predicting the extent of the pressure drop through the obstructed PHV. By investigating both normal and stenotic PHVs, normal ranges of velocity and turbulence level and the feasibility of 4D Flow MRI for pressure-drop prediction through the obstructed PHV were investigated.

\section{Methods}

In-vitro flow circuit and prosthetic heart valves

An in-vitro flow circuit was constructed to simulate valvular flow through the PHV (Figure 1a). PHVs were installed in a straight acrylic pipe with an inner diameter (D) of $26 \mathrm{~mm}$. The working fluid (tap water) was circulated through the flow circuit system under steady flow conditions by using a gear pump (ECO Gearchem G6, Pulsafeeder, NY). Three different flow rates (e.g., $6.7 \mathrm{~L} / \mathrm{min}$ ) were used to cover the range from mean cardiac output to peak flow rate in the ascending aorta (Table 1) [18]. The flow rate was controlled by changing the gear 
speed of the pump from 300 to 1200 revolutions per minute (RPM) and the exact flow rate was later confirmed from each set of 4D Flow MRI data. Two pressure ports were installed at $-5 \mathrm{D}$ and $+5 \mathrm{D}$ from the valve level (Figure 1a), and the pressure drop was directly measured using a digital pressure manometer (GMH-3161-07B, Greisinger, Germany).

Four different PHVs were studied: Medtronic-Hall tilting disc (Tilting disk; Medtronic Inc, Minneapolis, MN, USA), St. Jude Medical standard bileaflet (STJM; St. Jude Medical Inc, St Paul, MN, USA), Medtronic CoreValve Evolut R (Core; Medtronic, Minneapolis, MN, USA) and Edwards SAPIEN 3 (Edwards; Edwards Lifesciences Inc., Irvine, CA, USA) (Figure 2). To study abnormal hemodynamics in the PHVs, stenotic heart valves were artificially simulated. From a geometric point of view, a heart valve area less than $1.0 \mathrm{~cm}^{2}$ is defined as a severe stenosis $[19,20]$. Therefore, in this study, we artificially simulated the stenotic heart valves by restricting the opening areas of all tested valves less than $1.0 \mathrm{~cm}^{2}$. The leaflets of the Tilting disk and the STJM were glued to restrict the opening angle of the leaflet. The opening angle of the Tilting disk was restricted to $24^{\circ}$, providing an opening area of $0.39 \mathrm{~cm}^{2}$. One of the leaflets of the STJM was totally closed and another leaflet was restricted to $54^{\circ}$, providing an opening area of $0.59 \mathrm{~cm}^{2}$. Both leaflets of the CoreValve and the SAPIEN 3 were sutured so that around half of the leaflet commissure was closed, which made an opening area of $0.46 \mathrm{~cm}^{2}$ and $0.68 \mathrm{~cm}^{2}$, respectively. Opening areas of the valves were calculate from the face-views (Supplementary Figure 1)

\section{D Flow MRI measurement}

4D Flow MRI measurements were performed using a clinical 1.5T MRI scanner (1.5T Philips Achieva; Philips Medical Systems, Best, The Netherlands). A conventional gradient-echo sequence with asymmetric four-point flow encoding was modified to generate a six- 
directional icosahedral flow encoding (ICOSA6) and one flow-compensated reference encoding [13, 14, 21]. Four different velocity encoding ranges (VENCs) were used for each experimental condition: 1, 2, 3 and $5 \mathrm{~m} / \mathrm{s}$. Echo time (TE) and temporal resolution were 1.5$2.2 \mathrm{~ms}$ and $3.2-3.9 \mathrm{~ms}$, respectively. The flip angle was $10^{\circ}$. The field of view was $336 \mathrm{~mm} \times 336 \mathrm{~mm} \times 57 \mathrm{~mm}$ with a $1.5 \mathrm{~mm}$ isotropic voxel size. Partial echo with a factor of 0.7 along frequency-encoding directions was used to minimize TE, and the number of signal averages (NSA) was set to five to enhance the signal-to-noise ratio. Scan time for each measurement was about four minutes.

\section{D Flow MRI turbulence quantification}

For the conventional 4D Flow MRI, the MRI signal $S\left(k_{v}\right)$ for velocity distribution $s(v)$ is expressed by the Fourier transform as follows [22, 23]:

$$
S\left(k_{v}\right)=C \int_{-\infty}^{\infty} s(v) e^{-i k_{v} v} d v
$$

where $C$ is a constant scaling factor influenced by the relaxation parameter, spin density, receiver gain, etc. and $k_{v}$ is the amount of flow sensitivity, which is related to the velocity encoding parameter (VENC) as $k_{v}=\pi /$ VENC. When turbulent flow occurs in the region of interest, the intravoxel velocity variance (IVVV) of the turbulent flow along the $i$-direction, $\sigma_{i}^{2}$, can be estimated by the magnitude ratio between the reference signal without velocity encoding $S(0)$ and the signal with velocity encoding along the $i$-direction $S_{i}\left(k_{v}\right)$ as follows [22, 23]:

$$
\sigma_{i}^{2}=\frac{2}{k_{v}^{2}} \ln \left(\frac{\left|S_{i}(0)\right|}{\left|S_{i}\left(k_{v}\right)\right|}\right),(\mathrm{m} / \mathrm{s})
$$

Here, turbulence kinetic energy (TKE) of the flow can be calculated from IVVV in each direction as follows [24]: 


$$
T K E=\frac{1}{2} \rho \sum_{i=1}^{3} \sigma_{i}^{2},\left(\mathrm{~J} / \mathrm{m}^{3}\right)
$$

where $\rho$ is the density of the fluid.

When the velocity encoding direction is distributed to bisect the $2 \mathrm{D}$ plane in threedimensional Cartesian space (i.e., the $X Y, Y Z$ or $X Z$ plane), the obtained velocity and IVVV can be decomposed into two orthogonal components and its covariance term. Therefore, three velocity components ( $u, v$, and $w)$ and six elements of the Reynolds stress tensor $\mathrm{R}_{\mathrm{ij}}$ can be obtained by measuring six non-orthogonal velocity encodings and finding least square solutions for the six sets of directional phase and magnitude data [21].

Based on velocity and Reynolds stress obtained from the ICOSA6 sequence, turbulent production $P_{t}$ can be directly calculated as [14]:

$$
P_{t}=-R_{i j} S_{i j},(\mathrm{~J} / \mathrm{kg} \cdot \mathrm{s})
$$

where $S_{i j}$ are the strain rate tensors of mean velocity field. The subscripts $i$ and $j$ represent the perpendicular directions $x, y$ and $z$.

\section{Post-processing of 4D Flow MRI data}

Estimations of the three velocity components and Reynolds stress from 4D Flow MRI with ICOSA6 encoding were processed as described in previous publications [13, 14]. To extract the velocity of the flow, the data acquired with VENC $=5 \mathrm{~m} / \mathrm{s}$ were used. The velocity field obtained without flow was used to correct the velocity offsets caused by the background phase errors [25]. After the phase correction, peak velocity $V_{\text {peak }}$ was obtained by finding the maximum velocity in the flow region and the flow rate $Q$ was obtained by integrating the velocity at the cross-section of the flow.

Since four different VENC parameters from $1 \mathrm{~m} / \mathrm{s}$ to $5 \mathrm{~m} / \mathrm{s}$ were used, regardless of flow rate and valve, a multi-VENC approach was employed to sum the four datasets and 
optimize the sensitivity of the turbulence measurement [26]. At first, the Reynolds stress was reconstructed from the data obtained at VENC $=1 \mathrm{~m} / \mathrm{s}$. At any voxel of the data, if the flow encoded signal $S_{i}\left(k_{v}\right)$ was lower than three times the noise level, where the noise level was obtained from the standard deviation of the magnitude at the inlet flow, the corresponding Reynolds stress data were considered to be underestimated due to MRI noise [22]. Therefore, the Reynolds stress at the corresponding voxel was replaced with the data obtained with higher VENC $(2 \mathrm{~m} / \mathrm{s})$. If any voxel data obtained at VENC $=2 \mathrm{~m} / \mathrm{s}$ also showed $S_{i}\left(k_{v}\right)$ lower than three times noise, the Reynolds stress at the voxel was once again replaced with the data obtained with higher VENC (3 m/s). This process was continued until all Reynolds stress data were obtained from the lowest VENC measurement which provides $S_{i}\left(k_{v}\right)$ higher than three times the noise level.

To analyze the signal loss in the 4D Flow MRI due to the PHVs, signal intensity was normalized by the maximum intensity of the magnitude image. The normalized intensity of each PHV image was analyzed along the flow direction. The peak-to-peak length of local pit from the normalized intensity curve was measured to quantify the size of signal void.

\section{Bernoulli-based pressure drop estimation}

The following simplified Bernoulli equation has been widely used in medicine:

$$
\Delta \mathrm{P}_{\mathrm{SB}}=4 v_{\text {peak }}^{2},(m m H g)
$$

where $v_{\text {peak }}$ is the velocity at the vena contracta defined as the maximum velocity. More recently, the extended Bernoulli equation has been derived, which takes the pressure recovery at the post-stenosis into account [27]:

$$
\Delta P_{E B}=4 v_{\text {peak }}^{2}\left(1-\frac{E O A}{A_{A}}\right)^{2},(m m H g)
$$


where $E O A$ is the effective orifice area and $A_{A}$ is the area of the aorta. EOA can be obtained from the continuity equation assuming a flat axial velocity profile, $Q=E O A \cdot v_{\text {peak }}$, where $Q$ is the flow rate through the flow circuit.

\section{Turbulence-based pressure drop estimation}

Based on the work-energy balance in the flow pipe, the energy loss and the pressure drop of the flow can be expressed as follow [28]:

$$
\mathrm{E}=\mathrm{Q} \Delta \mathrm{P}(\mathrm{J} / \mathrm{s})
$$

where $Q$ is the flow rate and $\Delta P$ is the pressure drop of the flow. Assuming the energy loss of the turbulent flow is mostly dominated by turbulence production, $E$ can be estimated from the turbulence production. The product of the density and $P_{t}$ is the energy density of turbulence production with the unit of $\mathrm{J} / \mathrm{m}^{3} \cdot \mathrm{s}$ or $\mathrm{W} / \mathrm{m}^{3}$, and its volumetric integration results in total turbulence production within the region of interest with the unit of $\mathrm{W}$ or $\mathrm{mW}$.

$$
\mathrm{E}=\rho \int-R_{i j} S_{i j} d V=\rho \int P_{t} d V, \quad(\mathrm{~J} / \mathrm{s}, \mathrm{W})
$$

where $\rho$ is the density of the fluid and $d V$ is the volume of each voxel. Then, the pressure drop due to turbulence production can be expressed as [14]:

$$
\Delta \mathrm{P}_{T P}=\frac{\mathrm{E}}{\mathrm{Q}}=\frac{\rho}{Q} \int-R_{i j} S_{i j} d V
$$

The pressure drop $\Delta \mathrm{P}_{T P}$ was divided by the coefficient 133.32 to convert the unit of the pressure from Pa to mmHg.

\section{Statistical Analysis}

Linear regression was analyzed to assess the relationship between the predicted pressure drop from 4D Flow MRI and the measured pressure drop. Slope and coefficient of determination of 
the regression line $\left(R^{2}\right)$ were calculated using RStudio (RStudio, Inc., Boston, MA, USA). Bland-Altman analysis was also used to evaluate the agreement between the prediction and the measurement.

\section{Results}

The signal loss in the 4D Flow MRI varied with the type of PHV. When analyzing the normalized signal along the flow direction, the defect size of the signal from each valve was $37.5 \mathrm{~mm}, 39.0 \mathrm{~mm}, 37.5 \mathrm{~mm}$ and $51.0 \mathrm{~mm}$ for Tilting disk, STJM, SAPIEN 3 and CoreValve, respectively (Figure 3).

Peak velocities in the normal prosthetic valves were lower than $2 \mathrm{~m} / \mathrm{s}$. A flow rate of around $6.68-20.43 \mathrm{~L} / \mathrm{min}$ resulted in peak velocities of $0.49-1.14 \mathrm{~m} / \mathrm{s}, 0.74-1.01 \mathrm{~m} / \mathrm{s}, 0.70$ $1.34 \mathrm{~m} / \mathrm{s}$, and $0.71-1.11 \mathrm{~m} / \mathrm{s}$ for the Tilting disk, STJM, SAPIEN 3 and CoreValve, respectively (Figure 4 and Table 1). 4D Flow MRI clearly identified an increase in the peak velocity in the stenotic prosthetic valves (Table 1). Under the same flow rate conditions, the stenotic valves showed peak velocities of $1.47-4.17 \mathrm{~m} / \mathrm{s}, 1.53-4.31 \mathrm{~m} / \mathrm{s}, 1.38-2.49 \mathrm{~m} / \mathrm{s}$, and 1.02-1.72 m/s for the Tilting disk, STJM, SAPIEN 3 and CoreValve, respectively (Figure 4 and Table 1). While the Tilting disk, STJM and SAPIEN 3 generated high-velocity jet flow under the stenotic valve conditions, the jet-flow pattern could not be visually identified in the normal or stenotic CoreValve due to the relatively large signal void around the valve (Figure 4).

TKE in the normal PHVs were lower than 5.17 mJ. A flow rate of around 6.68-20.43 $\mathrm{L} / \mathrm{min}$ resulted in TKE values of $0.65-3.52 \mathrm{~mJ}, 0.62-4.12 \mathrm{~mJ}, 0.93-5.17 \mathrm{~mJ}$, and $0.84-5.04$ mJ for the Tilting disk, STJM, SAPIEN 3 and CoreValve, respectively (Figure 5 and Table 1). Under the same flow rate conditions, the stenotic valves increased TKE up to $25.53 \mathrm{~mJ}$. The 
stenotic PHVs showed TKE values of 3.2-25.53 mJ, 3.01-24.56 mJ, 2.2-12.33 mJ, and 0.97$5.59 \mathrm{~mJ}$ for the Tilting disk, STJM, SAPIEN 3 and CoreValve, respectively (Figure 5 and Table 1).

Total turbulence production in the normal PHVs was lower than $191.77 \mathrm{~mW}$, which is $559.38 \mathrm{~J} / \mathrm{m}^{3}$ when normalized by the flow rate. A flow rate of around $6.68-20.43 \mathrm{~L} / \mathrm{min}$ resulted in a total turbulence production of $58.62-168.55 \mathrm{~mW}, 46.56-118.17 \mathrm{~mW}, 59.69$ $191.77 \mathrm{~mW}$, and 58.94-169.08 $\mathrm{mW}$ for the Tilting disk, STJM, SAPIEN 3 and CoreValve, respectively (Figure 6 and Table 1). Under the same flow rate conditions, the stenotic valve increased the total turbulence production to $2139.28 \mathrm{~mW}$. The stenotic PHVs showed a total turbulence production of 157.35-2139.28 mW, 149.30-2069.41 mW, 114.71-655.85 mW, and 66.09-266.61 mW for the Tilting disk, STJM, SAPIEN 3 and CoreValve, respectively (Figure 6 and Table 1).

Pressure-drop estimations computed using simple and extended Bernoulli, normalized TKE (TKE/Q) and turbulence production were compared against experimentally measured pressure-drop values (Figure 7). Due to the large signal void of the CoreValve, all results deviated significantly from other valve data. Excluding the CoreValve, all predictions were linearly correlated to the measured pressure drop. The slope of the linear regression for the simplified Bernoulli was 1.17, and the Bland-Altman mean bias with 95\% limits of agreement was $8.95 \pm 13.45 \mathrm{mmHg}$. The slope of the linear regression for the extended Bernoulli was 0.87, and the Bland-Altman mean bias with $95 \%$ limits of agreement was $-0.21 \pm 8.54 \mathrm{mmHg}$. The slope of the linear regression for the turbulence production method was 0.75 , and the Bland-Altman mean bias with 95\% limits of agreement was $-0.21 \pm 10.87 \mathrm{mmHg}$. The slope of the linear regression for TKE/Q was $0.99\left(\mathrm{R}^{2}=0.986, \mathrm{p}<0.001\right)$ 


\section{Discussion}

This study aimed to assess the valvular flow characteristics in different PHVs using 4D Flow MRI and to investigate the feasibility of using 4D Flow MRI measurement to predict the elevation of the pressure drop through stenotic PHVs. The major findings of this study were that: (1) normal ranges of turbulence levels, including TKE and turbulence production in a range of PHVs, were characterized using 4D Flow MRI, (2) 4D Flow MRI pressure-drop predictions based on both Bernoulli-based and turbulence-based methods were found to correlate well with the elevation of the pressure drop through the majority of the stenotic PHVs, (3) in contrast to the Tilting disc, STJM and SAPIEN 3, the obstructive hemodynamics in the stenotic CoreValve were not clearly identified using 4D Flow MRI due to the large signal void from the long strut, resulting in pressure predictions using 4D Flow MRI which deviated from the true pressure values.

It has been widely known that the velocity fluctuation in the turbulent flow results in signal loss of MRI image [29-32]. Mechanisms involved in the reduction of the signal magnitude due to velocity fluctuations include ghosting and intravoxel phase dispersion. Intravoxel phase dispersion is caused by the distribution of spin velocities within a voxel, which results in destructive interference of signal phases. Of the mechanisms involved in creating signal loss due to velocity fluctuations, intravoxel phase dispersion has been shown to be the dominant source $[30,33]$. Previous studies have derived expressions that describe the effects of velocity fluctuations on the MRI signal in order to develop methods for the quantification of turbulence intensity [34-40]. Assuming that the phase of the MRI signal is Gaussian distributed in turbulent flow, Gao and Gore derived a relationship between the MRI signal and the turbulence intensity [38]. Recently, as the advancement of 4D Flow MRI facilitates investigations of the hemodynamics of cardiovascular blood flow under in-vivo 
conditions, the turbulence quantification using 4D Flow MRI has been widely used to characterize the turbulent cardiovascular flows [22, 41-44].

One of the main questions of this study was: can 4D Flow MRI predict the pressure drop through prosthetic valves despite the signal void around the prosthetic valves? While 4D Flow MRI provides multiple methods for pressure-drop estimation, those non-invasive estimations are mostly based on velocity and turbulence measurements, which can be biased when the metal components of the prosthetic valves prevent accurate measurements near the valve. In this study, the 4D flow-based estimations of the elevation of the pressure drop through the stenotic Tilting disk, STJM and SAPIEN 3 valves correlated well with the true values. This indicates that $4 \mathrm{D}$ Flow MRI assessments of the velocity and turbulence are accurate despite signal void regions extending 15 to $20 \mathrm{~mm}$ from the valve. This result seems to agree with previous studies on peak velocity and total turbulence quantification using 4D Flow MRI [14]. According to previous studies, the velocity of the jet flow usually reaches a maximum at around $10 \mathrm{~mm}$ distal to the valve as the flow converges at the post-valve region, and retains more than $85 \%$ of the peak velocity at $30 \mathrm{~mm}$ distal to the valve [45]. Meanwhile, turbulence production usually occurs distal to the valve, reaching a maximum at the aortic root and ascending aorta [14, 42, 46, 47]. Considering that the length of signal loss behind the outlet orifice of the valve is less than half of the total signal void of the valves (Figure 3), the Tilting disc, STJM and SAPIEN 3 valves should be relatively free from significant underestimation of peak velocity and turbulence production. In cfontrast, the large strut of the CoreValve and the corresponding signal void were found to prevent accurate measurements of the velocity and turbulence production. Although the stenotic CoreValve had a smaller orifice area than that of the SAPIEN 3 valve, generating a higher pressure drop, 4D Flow MRI provided lower peak velocity, TKE and turbulence production even than those of the SAPIEN 
3 valve. Therefore, a 4D Flow MRI prediction of the pressure drop through the CoreValve was not feasible.

Understanding detailed velocity and turbulence characteristics in PHVs are important issues for improving the hemodynamic performance of current PHVs. Although they have proven benefits and durability, PHVs are associated with several complications, including thromboembolism, prosthesis-patient mismatch, structural valve dysfunction, endocarditis, and hemolysis. Recently, several reports have highlighted an increased incidence of thrombus formation and reduced leaflet motion with TAVI valves [48-50]. These findings were widely subclinical since the pressure gradient based on TEE was not increased, and the diagnosis was made using ECG-gated computer tomography. However, a large proportion of these complications are related to abnormal hemodynamics induced by the PHV [3]. 4D Flow MRI has the potential to improve the diagnostics of stenotic prosthetic valves, and our findings form the basis for future prospective clinical studies. In this study, we have summarized the hemodynamic performances of a range of PHVs under various flow conditions. These valvespecific data should be of great interest from basic science, clinical, and industrial points of view, especially with respect to developing novel PHVs [9].

Although the Bernoulli method worked well in the present experiments, there are some scenarios in which the turbulence method can be expected to complement the Bernoulli method. First, since the Bernoulli method is based on the conventional sudden contraction/expansion flow phenomena [51], it is expected to perform less well in scenarios with multiple stenoses and multiple orifices. Recent study showed that the prosthetic valves with multiple jets resulted in larger pressure drops compared to the single jet condition while the Bernoulli method predicts the similar pressure drops for both conditions [52]. Second, since the Bernoulli method assumes inviscid fluid and negligible viscous effects, it may be 
less accurate for low flow with relatively high viscous effects, such as paradoxical low-flow, low-gradient severe aortic stenosis [53]. Since the turbulence production method does not assume the inviscid fluid condition, it may help identify severe stenosis at a low flow condition. Therefore, the turbulence-based method may have advantages for the assessment of stenoses in various clinical conditions. However, we acknowledge that none of these scenarios were demonstrated in the current study and remain to be explored in future studies.

It should also be noted that turbulence not only can indicate the possible pressure drop of the blood flow, but also the turbulence-induced hemodynamic blood damage [54] and nearwall disturbance on the vessel [55]. Therefore, turbulence quantification gives more clinical information than conventional Bernoulli-based methods. The ICOSA6 sequence used in this study can provide both velocity and turbulence with a single VENC measurement [56]. Therefore, turbulence quantification would not need multiple VENC measurements. In this study, we used two different VENC for the velocity and turbulence quantification to eliminate any possible phase-unwrapping issues, which is beyond the scope of this study.

One of the limitations of this study is that the effects of pulsatile flow have not been examined. The use of steady flow reduces the complexity of the experiments, allowing the examination of other desired parameters, such as effect of the prosthetic valve type and flow rate. Furthermore, steady flow removes the complexity of choosing proper Bernoulli equation, pressure drop measurement technique and temporal resolution related to the pulsatile flow measurements. In this study, we aimed to study if 4D Flow MRI can provide valvular flow characteristics and pressure drop in a variety of normal and stenotic prosthetic heart valves (PHVs). The flow characteristics and pressure drop of the stenotic PHVs were mostly determined by the opening angle and the corresponding opening area of the PHVs [52, 57]. Therefore, we prioritized to investigate the steady flow conditions with the fixed opening 
angles of the PHVs. As this study demonstrates the feasibility of 4D Flow MRI for quantification of normal and stenotic blood flow in PHVs, future studies should consider the effects of the pulsatility of the flow, elasticity of the vessel and temporal resolution.

Flow pulsatility in the non-rigid blood vessel induces temporal changes of kinetic energy within the fluid volume and flux of the kinetic energy at the fluid entrance and exit. Therefore, a turbulence-based method for pulsatile flow measurement requires considering the change in kinetic energy, taking into account the flow acceleration and deceleration [14]. Given that the kinetic energy can be easily obtained from the 4D Flow MRI [58], the application of the present method in physiological pulsatile flows would be straightforward.

The turbulence-based method used in this study assumes that the pressure drop through the PHV is mostly induced by the turbulence production at the PHV. The current method does not include any other loss due to vessel friction, curvature and branching. Therefore, the turbulence-based estimation of the pressure drop for the in-vivo conditions may be underestimated if additional sources of loss are not negligible. Therefore, the turbulence-based method is not recommended to be used to measure the pressure drop in small arteries where laminar flows develop.

We also note that the measurement setup used for this study is optimized for steady flow measurement. The application of the methods for in-vivo conditions with pulsatile flow would require some adjustments in the acquisition protocol. Compared to conventional 4D flow MRI acquisitions, the ICOSA6 4D flow MRI used in this study results in an about 75\% increase in acquisition time related to the increased number of flow encoding directions. However, the ICOSA6 measurement allows for improved turbulent quantification, allowing for computation of turbulent production, and is less sensitive to phase wraps [59], thus avoiding the need for dual-VENC acquisition. Otherwise, in-vivo acquisition and post 
processing, including background phase correction, of ICOSA6 4D flow MRI is in principle similar to conventional 4D flow MRI, which has been extensively discussed in the 4D flow MRI consensus statement [60].

In this study, all measurements were performed at 1.5T MRI. It is noted that 1.5T MRI in general produces less metal artifacts due to less metal-induced field inhomogeneity compared to 3.0T MRI [61, 62]. Therefore, the size of signal void due to the PHVs and corresponding loss of the flow data would be larger if they were measured with 3.0T MRI.

It is also noted that all pressure drop values in this study are relative to the pressure at the reference point. The method does not provide any absolute pressure values. In addition, all pressure drop estimations in this study did not include data from the signal void, therefore, all pressure drop estimations implicitly assume that no pressure loss was induced within the signal void.

In this study, all experiments were performed with water as working fluid. The flow rates ranged from around 6.46 to $20.95 \mathrm{~L} / \mathrm{min}$, which corresponds to the Reynolds number of 5,262 to 17,065. Although the use of water resulted in a higher Reynolds number, this range still covers the Reynolds number of aortic blood flow of around 5,000 to 10,000 [63-65]. In addition, the aortic flow belongs to the highly turbulent flow regime [66], where the viscosity effect is negligible. Also the signal void will to some extent depend on the used working fluid. The relative differences are expected to be small.

According to the previous study [14], the quantification of the turbulence production was not substantially influenced by SNR, resulting in less than $2 \%$ mean bias at signal-tonoise ratio $(\mathrm{SNR})>10$. Considering that conventional 4D Flow MRI acquisitions have SNR $>30$ without the use of a contrast agent [67], the influence of SNR on the turbulence production in practical 4D Flow MRI measurements will not cause substantial errors. 
In addition, the test model used in this study was neither anatomically shaped nor compliant. Future work will be required to examine hemodynamics in PHVs under more physiological circumstances, in vitro, or in vivo. Furthermore, we have studied four different PHVs. The Tilting disc valve, which is no longer implanted in the western world, was included in our experiment since a large body of experimental data has shown velocity and turbulence level in this valve [68-70]. A previous study showed that total TKE of $0.9 \mathrm{~mJ}$ for tilting-disc and $1.2 \mathrm{~mJ}$ for STJM at the flow rate of $12 \mathrm{~L} / \mathrm{min}$. In this study, maximum total TKE for the normal tilting-disc and STJM were $0.65-3.52 \mathrm{~mJ}$ and $0.62-4.12 \mathrm{~mJ}$, respectively (Table 1). These results show that normal PHVs can develop total TKE less than $5 \mathrm{~mJ}$ [70]. Even though the specific data are limited to these prostheses, we have demonstrated the feasibility of 4D Flow MRI to assess normal and stenotic PHVs.

Compared that this study, which used 4D Flow MRI to directly measure the blood flow and estimate pressure drops, numerical simulation such as computational fluid dynamics (CFD) has also been widely used to investigate hemodynamics in the patient-specific model. Previous studies showed that high resolution flow data from CFD can be useful to diagnose the abnormal hemodynamics in various types of the blood vessels [68, 69, 71]. Another advantage of CFD has that it can be used to perform virtual planning of surgical repairs or interventions in patients with congenital heart diseases [72]. Unfortunately, numerical simulations depend heavily on the geometry and inflow and outflow conditions, which are not always possible to obtain with sufficient quality. A recent study successfully compared the intracardiac blood flow obtained from both 4D Flow MRI and CFD [73], which opens up for combining the unique advantages of 4D Flow MRI measurement and CFD.

In conclusion, 4D Flow MRI assessment is feasible for many normal and stenotic prosthetic heart valves despite significant signal voids. Of the valves evaluated in this study, 
the Tilting disc, STJM and SAPIEN 3 valves were shown to be relatively free from significant underestimation of peak velocity, turbulence production and corresponding pressure-drop estimation. In contrast, the large strut of the CoreValve and the corresponding signal void prevented accurate measurements of the velocity and turbulence production; therefore, 4D Flow MRI prediction of the pressure drop through the CoreValve was not feasible.

\section{Acknowledgments}

We thank Federica Viola for providing valuable advice related to the 4D Flow MRI measurements.

\section{Source of Funding}

The research leading to these results has received funding from the European Union's Seventh Framework Programme (FP7/2007-2013) under grant agreement 310612 and the Swedish Research Council; Grant numbers: 2013-6077 and 2014-6191. This research was supported by the Basic Science Research Program through the National Research Foundation of Korea (NRF), funded by the Ministry of Education (2018R1D1A1A02043249).

\section{Disclosures}

The authors declare no competing financial interests.

\section{References}

[1] Pibarot P, Dumesnil JG. Prosthetic heart valves. Circulation 2009;119(7):1034-48.

[2] Richau J, Dieringer MA, Traber J, von Knobelsdorff-Brenkenhoff F, Greiser A, Schwenke C, et al. Effects of heart valve prostheses on phase contrast flow measurements in Cardiovascular Magnetic Resonance-a phantom study. Journal of Cardiovascular Magnetic Resonance 2017;19(1):5.

[3] Vesey JM, Otto CM. Complications of prosthetic heart valves. Current cardiology reports 2004;6(2):106-11. 
[4] Leetmaa T, Hansson NC, Leipsic J, Jensen K, Poulsen SH, Andersen HR, et al. Early Aortic Transcatheter Heart Valve Thrombosis. Circulation: Cardiovascular Interventions 2015;8(4):e001596.

[5] Cawley PJ, Maki JH, Otto CM. Cardiovascular magnetic resonance imaging for valvular heart disease. Circulation 2009;119(3):468-78.

[6] Myerson SG. Heart valve disease: investigation by cardiovascular magnetic resonance. Journal of Cardiovascular Magnetic Resonance 2012;14(1):7.

[7] Niederberger J, Schima H, Maurer G, Baumgartner H. Importance of pressure recovery for the assessment of aortic stenosis by Doppler ultrasound. Circulation 1996;94(8):1934-40.

[8] Vandervoort PM, Greenberg NL, Pu M, Powell KA, Cosgrove DM, Thomas JD. Pressure recovery in bileaflet heart valve prostheses. Circulation 1995;92(12):3464-72.

[9] Kvitting JPE, Dyverfeldt P, Sigfridsson A, Franzén S, Wigström L, Bolger AF, et al. In vitro assessment of flow patterns and turbulence intensity in prosthetic heart valves using generalized phase-contrast MRI. Journal of Magnetic Resonance Imaging 2010;31(5):1075-80.

[10] Caruthers SD, Lin SJ, Brown P, Watkins MP, Williams TA, Lehr KA, et al. Practical value of cardiac magnetic resonance imaging for clinical quantification of aortic valve stenosis. Circulation 2003;108(18):2236-43.

[11] Donati F, Myerson S, Bissell MM, Smith NP, Neubauer S, Monaghan MJ, et al. Beyond Bernoulli. Circulation: Cardiovascular Imaging 2017;10(1):e005207.

[12] Falahatpisheh A, Rickers C, Gabbert D, Heng EL, Stalder A, Kramer HH, et al. Simplified Bernoulli's method significantly underestimates pulmonary transvalvular pressure drop. Journal of Magnetic Resonance Imaging 2016;43(6):1313-9.

[13] Ha H, Lantz J, Haraldsson H, Casas B, Ziegler M, Karlsson M, et al. Assessment of turbulent viscous stress using ICOSA 4D Flow MRI for prediction of hemodynamic blood damage. Scientific reports 2016;6.

[14] Ha H, Lantz J, Ziegler M, Casas B, Karlsson M, Dyverfeldt P, et al. Estimating the irreversible pressure drop across a stenosis by quantifying turbulence production using 4D Flow MRI. Scientific Reports 2017;7.

[15] Oechtering TH, Hons CF, Sieren M, Hunold P, Hennemuth A, Huellebrand M, et al. Timeresolved 3-dimensional magnetic resonance phase contrast imaging (4D Flow MRI) analysis of hemodynamics in valve-sparing aortic root repair with an anatomically shaped sinus prosthesis. The Journal of thoracic and cardiovascular surgery 2016;152(2):418-27. e1.

[16] Maragiannis D, Jackson M, Igo S, Chin K, Autry K, Ghosn MG, et al. Bioprosthetic mitral valve effective orifice area using 4D flow cardiac magnetic resonance derived time velocity integral. An in vitro comparison with Doppler Echocardiography. Journal of Cardiovascular Magnetic Resonance 2015;17(S1):P336.

[17] Giese D, Weiss K, Baeßler B, Madershahian N, Choi Y-H, Maintz D, et al. In vitro evaluation of flow patterns and turbulent kinetic energy in trans-catheter aortic valve prostheses. Magnetic Resonance Materials in Physics, Biology and Medicine 2018;31(1):165-72.

[18] Powell A, Maier S, Chung T, Geva T. Phase-velocity cine magnetic resonance imaging measurement of pulsatile blood flow in children and young adults: in vitro and in vivo validation. Pediatric cardiology 2000;21(2):104-10.

[19] Members: ATF, Falk V, Baumgartner H, Bax JJ, De Bonis M, Hamm C, et al. 2017 ESC/EACTS Guidelines for the management of valvular heart disease. European journal of cardio-thoracic surgery 2017;52(4):616-64.

[20] Bonow RO, Brown AS, Gillam LD, Kapadia SR, Kavinsky CJ, Lindman BR, et al. ACC/AATS/AHA/ASE/EACTS/HVS/SCA/SCAI/SCCT/SCMR/STS 2017 appropriate use criteria for the treatment of patients with severe aortic stenosis: a report of the American College of Cardiology Appropriate Use Criteria Task Force, American Association for Thoracic Surgery, American Heart Association, American Society of Echocardiography, European Association for Cardio-Thoracic Surgery, Heart Valve Society, Society of Cardiovascular Anesthesiologists, Society for Cardiovascular Angiography and Interventions, Society of Cardiovascular Computed 
Tomography, Society for Cardiovascular Magnetic Resonance, and Society of Thoracic Surgeons. Journal of the American College of Cardiology 2017:24131.

[21] Haraldsson H, Kefayati S, Ahn S, Dyverfeldt P, Lantz J, Karlsson M, et al. Assessment of Reynolds stress components and turbulent pressure loss using 4D flow MRI with extended motion encoding. Magnetic Resonance in Medicine 2017.

[22] Dyverfeldt P, Gårdhagen R, Sigfridsson A, Karlsson M, Ebbers T. On MRI turbulence quantification. Magnetic resonance imaging 2009;27(7):913-22.

[23] Dyverfeldt P, Sigfridsson A, Kvitting JPE, Ebbers T. Quantification of intravoxel velocity standard deviation and turbulence intensity by generalizing phase-contrast MRI. Magnetic resonance in medicine 2006;56(4):850-8.

[24] Pope SB. Turbulent flows. IOP Publishing; 2001.

[25] Petersson S, Dyverfeldt P, Sigfridsson A, Lantz J, Carlhäll CJ, Ebbers T. Quantification of turbulence and velocity in stenotic flow using spiral three-dimensional phase-contrast MRI. Magnetic resonance in medicine 2016;75(3):1249-55.

[26] Ha H, Kim GB, Kweon J, Kim YH, Kim N, Yang DH, et al. Multi-VENC acquisition of fourdimensional phase-contrast MRI to improve precision of velocity field measurement. Magnetic resonance in medicine 2016;75(5):1909-19.

[27] Garcia D, Pibarot P, Dumesnil JG, Sakr F, Durand L-G. Assessment of aortic valve stenosis severity a new index based on the energy loss concept. Circulation 2000;101(7):765-71.

[28] Winter H. Viscous dissipation term in energy equations. Calculation and Measurement Techniques for Momentum, Energy and Mass Transfer 1987;7:27-34.

[29] Evans AJ, Blinder RA, Herfkens RJ, Spritzer CE, Kuethe DO, Fram EK, et al. Effects of turbulence on signal intensity in gradient echo images. Investigative radiology 1988;23(7):512-8.

[30] Gatenby J, McCauley T, Gore J. Mechanisms of signal loss in magnetic resonance imaging of stenoses. Medical physics 1993;20(4):1049-57.

[31] Oshinski JN, Ku DN, Pettigrew RI. Turbulent fluctuation velocity: the most significant determinant of signal loss in stenotic vessels. Magnetic resonance in medicine 1995;33(2):193-9.

[32] Urchuk SN, Plewes DB. Mechanisms of flow-induced signal loss in MR angiography. Journal of Magnetic Resonance Imaging 1992;2(4):453-62.

[33] Siegel Jr JM, Oshinski JN, Pettigrew RI, Ku DN. Computational simulation of turbulent signal loss in 2D time-of-flight magnetic resonance angiograms. Magnetic resonance in medicine 1997;37(4):609-14.

[34] De Gennes P. Theory of spin echoes in a turbulent fluid. Physics Letters A 1969;29(1):20-1.

[35] Fukuda K, Hirai A. A pulsed NMR study on the flow of fluid. Journal of the Physical Society of Japan 1979;47(6):1999-2006.

[36] Xiang Q, Nalcioglu O. Measurement of mean and variance of velocity fields within each voxel by NMR imaging. IEEE transactions on medical imaging 1988;7(4):364-7.

[37] Kuethe DO. Measuring distributions of diffusivity in turbulent fluids with magnetic-resonance imaging. Physical Review A 1989;40(8):4542.

[38] Gao JH, Gore JC. Turbulent flow effects on NMR imaging: measurement of turbulent intensity. Medical physics 1991;18(5):1045-51.

[39] Gatenby J, Gore J. Mapping of turbulent intensity by magnetic resonance imaging. Journal of Magnetic Resonance, Series B 1994;104(2):119-26.

[40] Kuethe DO, Gao J-H. NMR signal loss from turbulence: models of time dependence compared with data. Physical Review E 1995;51(4):3252.

[41] Dyverfeldt P, Sigfridsson A, Kvitting JPE, Ebbers T. Quantification of intravoxel velocity standard deviation and turbulence intensity by generalizing phase-contrast MRI. Magnetic Resonance in Medicine: An Official Journal of the International Society for Magnetic Resonance in Medicine 2006;56(4):850-8.

[42] Dyverfeldt P, Hope MD, Tseng EE, Saloner D. Magnetic resonance measurement of turbulent kinetic energy for the estimation of irreversible pressure loss in aortic stenosis. JACC: Cardiovascular Imaging 2013;6(1):64-71. 
[43] Dyverfeldt P, Kvitting JPE, Sigfridsson A, Engvall J, Bolger AF, Ebbers T. Assessment of fluctuating velocities in disturbed cardiovascular blood flow: in vivo feasibility of generalized phase-contrast MRI. Journal of Magnetic Resonance Imaging: An Official Journal of the International Society for Magnetic Resonance in Medicine 2008;28(3):655-63.

[44] Ha H, Kim GB, Kweon J, Huh HK, Lee SJ, Koo HJ, et al. Turbulent kinetic energy measurement using phase contrast MRI for estimating the post-stenotic pressure drop: in vitro validation and clinical application. PloS one 2016;11(3):e0151540.

[45] Garcia J, Marrufo OR, Rodriguez AO, Larose E, Pibarot P, Kadem L. Cardiovascular magnetic resonance evaluation of aortic stenosis severity using single plane measurement of effective orifice area. Journal of Cardiovascular Magnetic Resonance 2012;14(1):23.

[46] Dyverfeldt P, Kvitting JPE, Sigfridsson A, Engvall J, Bolger AF, Ebbers T. Assessment of fluctuating velocities in disturbed cardiovascular blood flow: In vivo feasibility of generalized phase-contrast MRI. Journal of Magnetic Resonance Imaging 2008;28(3):655-63.

[47] Kweon J, Yang DH, Kim GB, Kim N, Paek M, Stalder AF, et al. Four-dimensional flow MRI for evaluation of post-stenotic turbulent flow in a phantom: comparison with flowmeter and computational fluid dynamics. European radiology 2016;26(10):3588-97.

[48] Testa L, Latib A. Assessing the Risk of Leaflet Motion Abnormality Following Transcatheter Aortic Valve Implantation. Interventional Cardiology Review 2018;13(1):37.

[49] Sondergaard L, De Backer O, Kofoed KF, Jilaihawi H, Fuchs A, Chakravarty T, et al. Natural history of subclinical leaflet thrombosis affecting motion in bioprosthetic aortic valves. European heart journal 2017;38(28):2201-7.

[50] Makkar RR, Fontana G, Jilaihawi H, Chakravarty T, Kofoed KF, De Backer O, et al. Possible subclinical leaflet thrombosis in bioprosthetic aortic valves. New England Journal of Medicine 2015;373(21):2015-24.

[51] Garcia D, Pibarot P, Dumesnil JG, Sakr F, Durand L-G. Assessment of aortic valve stenosis severity: a new index based on the energy loss concept. Circulation 2000;101(7):765-71.

[52] Ha H, Koo HJ, Huh HK, Kim GB, Kweon J, Kim N, et al. Effect of pannus formation on the prosthetic heart valve: In vitro demonstration using particle image velocimetry. PloS one 2018;13(6):e0199792.

[53] Baumgartner H. Low-flow, low-gradient aortic stenosis with preserved ejection fraction. Journal of the American College of Cardiology 2012;60(14):1268-70.

[54] Ha H, Lantz J, Haraldsson H, Casas B, Ziegler M, Karlsson M, et al. Assessment of turbulent viscous stress using ICOSA 4D Flow MRI for prediction of hemodynamic blood damage. Scientific Reports 2016;6:39773.

[55] Ziegler M, Lantz J, Ebbers T, Dyverfeldt P. Assessment of turbulent flow effects on the vessel wall using four-dimensional flow MRI. Magnetic resonance in medicine 2017;77(6):2310-9.

[56] Haraldsson H, Kefayati S, Ahn S, Dyverfeldt P, Lantz J, Karlsson M, et al. Assessment of Reynolds stress components and turbulent pressure loss using 4D flow MRI with extended motion encoding. Magnetic resonance in medicine 2018;79(4):1962-71.

[57] Koo HJ, Ha H, Kang J-W, Kim JA, Song J-K, Kim HJ, et al. Impact of pannus formation on hemodynamic dysfunction of prosthetic aortic valve: pannus extent and its relationship to prosthetic valve motion and degree of stenosis. Clinical Research in Cardiology 2018:1-11.

[58] Donati F, Myerson S, Bissell MM, Smith NP, Neubauer S, Monaghan MJ, et al. Beyond BernoulliCLINICAL PERSPECTIVE. Circulation: Cardiovascular Imaging 2017;10(1):e005207.

[59] Zwart NR, Pipe JG. Multidirectional high-moment encoding in phase contrast MRI. Magnetic resonance in medicine 2013;69(6):1553-63.

[60] Dyverfeldt P, Bissell M, Barker AJ, Bolger AF, Carlhäll C-J, Ebbers T, et al. 4D flow cardiovascular magnetic resonance consensus statement. Journal of Cardiovascular Magnetic Resonance 2015;17(1):72.

[61] Radzi S, Cowin G, Robinson M, Pratap J, Volp A, Schuetz MA, et al. Metal artifacts from titanium and steel screws in CT, 1.5 T and 3T MR images of the tibial Pilon: a quantitative assessment in 3D. Quantitative imaging in medicine and surgery 2014;4(3):163. 
[62] Olsrud J, Lätt J, Brockstedt S, Romner B, Björkman-Burtscher IM. Magnetic resonance imaging artifacts caused by aneurysm clips and shunt valves: dependence on field strength (1.5 and $3 \mathrm{~T}$ ) and imaging parameters. Journal of Magnetic Resonance Imaging: An Official Journal of the International Society for Magnetic Resonance in Medicine 2005;22(3):433-7.

[63] Stein PD, Sabbah HN. Turbulent blood flow in the ascending aorta of humans with normal and diseased aortic valves. Circulation research 1976;39(1):58-65.

[64] Gülan U, Binter C, Kozerke S, Holzner M. Shear-scaling-based approach for irreversible energy loss estimation in stenotic aortic flow-an in vitro study. Journal of biomechanics 2017;56:89-96.

[65] Stalder AF, Frydrychowicz A, Russe MF, Korvink JG, Hennig J, Li K, et al. Assessment of flow instabilities in the healthy aorta using flow-sensitive MRI. Journal of Magnetic Resonance Imaging 2011;33(4):839-46.

[66] Pope S, Pope SB. Turbulent Flows. Cambridge University Press; 2000.

[67] Bock J, Frydrychowicz A, Stalder AF, Bley TA, Burkhardt H, Hennig J, et al. 4D phase contrast MRI at 3 T: Effect of standard and blood-pool contrast agents on SNR, PC-MRA, and blood flow visualization. Magnetic Resonance in Medicine: An Official Journal of the International Society for Magnetic Resonance in Medicine 2010;63(2):330-8.

[68] Yoganathan AP, Woo Y-R, Sung H-W. Turbulent shear stress measurements in the vicinity of aortic heart valve prostheses. Journal of biomechanics 1986;19(6):433-42.

[69] Yoganathan AP, Sung H, Woo Y, Jones M. In vitro velocity and turbulence measurements in the vicinity of three new mechanical aortic heart valve prostheses: Björk-Shiley Monostrut, OmniCarbon, and Duromedics. The Journal of thoracic and cardiovascular surgery 1988;95(5):929-39.

[70] Kvitting JPE, Dyverfeldt P, Sigfridsson A, Franzén S, Wigström L, Bolger AF, et al. In vitro assessment of flow patterns and turbulence intensity in prosthetic heart valves using generalized phase-contrast MRI. Journal of Magnetic Resonance Imaging: An Official Journal of the International Society for Magnetic Resonance in Medicine 2010;31(5):1075-80.

[71] Yun BM, Dasi L, Aidun C, Yoganathan A. Highly resolved pulsatile flows through prosthetic heart valves using the entropic lattice-Boltzmann method. Journal of Fluid Mechanics 2014;754:122-60.

[72] Baretta A, Corsini C, Yang W, Vignon-Clementel IE, Marsden AL, Feinstein JA, et al. Virtual surgeries in patients with congenital heart disease: a multi-scale modelling test case. Phil Trans $\mathrm{R}$ Soc A 2011;369(1954):4316-30.

[73] Lantz J, Gupta V, Henriksson L, Karlsson M, Persson A, Carlhäll C-J, et al. Intracardiac flow at 4D CT: comparison with 4D flow MRI. Radiology 2018:173017. 


\section{Figure Legends}

Figure 1. Schematics of in-vitro experiments. (a) fluid-circuit system and (b) mechanical heart valves and TAVI valves used for this study.

Figure 2. Prosthetic valves used in this study. (a) normal Edwards SAPIEN 3 (b) normal CoreValve Evolut R, (c) stenotic Edwards SAPIEN3 (d) stenotic CoreValve Evolut R, (e) stenotic tilted disc valve, (f) stenotic St. Jude mechanical valve, (g) side-view of stenotic tilted disc valve, (h) side-view of stenotic St. Jude mechanical valve.

Figure 3. Signal loss in MRI signal due to the prosthetic valves. (a) magnitude images, (b) normalized signal analysis.

Figure 4. Comparison of the velocity field in various prosthetic valves under normal and stenotic conditions. Representative cases with a flow rate of $\sim 20.43 \mathrm{~L} / \mathrm{min}$ are shown. While dashed lines indicate the direction of the valve leaflets.

Figure 5. Comparison of the turbulent kinetic energy (TKE) in various prosthetic valves under normal and stenotic conditions. Representative cases with a flow rate of $\sim 20.43 \mathrm{~L} / \mathrm{min}$ are shown.

Figure 6. Comparison of turbulence production in various prosthetic valves under normal and stenotic conditions. Representative cases with a flow rate of $\sim 20.43 \mathrm{~L} / \mathrm{min}$ are shown.

Figure 7. Linear regression analysis of 4D Flow MRI pressure drop estimations. Linear regression between the measured pressure drop and 4D Flow MRI predictions using (a) simplified Bernoulli, (b) extended Bernoulli, (c) normalized TKE (TKE/Q) and (d) turbulence production. 
Table 1. Summary of the in-vitro experiments

\begin{tabular}{|c|c|c|c|c|c|c|c|c|c|c|c|c|c|}
\hline Stenosis & $\begin{array}{l}\text { Valve } \\
\text { Type }\end{array}$ & $\begin{array}{c}\mathbf{Q} \\
{[\mathbf{L} / \mathbf{m i n}]}\end{array}$ & $\begin{array}{c}\text { Reynolds } \\
\text { number }\end{array}$ & $\begin{array}{c}\text { Peak } \\
\text { Velocity } \\
{[\mathrm{m} / \mathrm{s}]} \\
\end{array}$ & $\begin{array}{c}\text { EOA } \\
{\left[\mathbf{m}^{2}\right]}\end{array}$ & $\begin{array}{c}\text { Turbulence } \\
\text { Production } \\
{[\mathrm{mW}]}\end{array}$ & $\begin{array}{c}\text { Turbulence } \\
\text { Production/Q } \\
{\left[\mathbf{J} / \mathbf{m}^{3}\right]} \\
\end{array}$ & $\begin{array}{l}\text { TKE } \\
{[\mathrm{mJ}]}\end{array}$ & $\begin{array}{l}\text { TKE/Q } \\
{\left[\mathbf{J} \text { s/m } \mathbf{m}^{3}\right]}\end{array}$ & $\begin{array}{c}\mathbf{d P}_{\text {meas }} \\
{[\mathrm{mmHg}]}\end{array}$ & $\begin{array}{c}\text { dPsB } \\
{\left[\mathrm{mmHg}_{\mathrm{S}}\right.} \\
]\end{array}$ & $\begin{array}{c}\mathrm{dP}_{\mathrm{EB}} \\
{[\mathrm{mmHg}]}\end{array}$ & $\begin{array}{c}\mathbf{d P}_{\mathrm{TP}} \\
{[\mathrm{mmHg}]}\end{array}$ \\
\hline \multirow{12}{*}{$\begin{array}{l}\text { Normal } \\
\text { valve }\end{array}$} & \multirow{3}{*}{$\begin{array}{l}\text { Tilting } \\
\text { disc }\end{array}$} & 6.82 & 5555 & 0.49 & 2.33 & 58.62 & 515.52 & 0.65 & 5.69 & \multirow{12}{*}{ NA } & 0.95 & 0.30 & 3.87 \\
\hline & & 13.55 & 11037 & 0.88 & 2.56 & 96.19 & 425.80 & 1.80 & 7.95 & & 3.11 & 0.83 & 3.19 \\
\hline & & 20.61 & 16788 & 1.14 & 3.01 & 168.55 & 490.74 & 3.52 & 10.26 & & 5.22 & 0.98 & 3.68 \\
\hline & \multirow{3}{*}{ STJM } & 6.79 & 5531 & 0.74 & 1.52 & 46.56 & 411.71 & 0.62 & 5.48 & & 2.21 & 1.12 & 3.09 \\
\hline & & 13.92 & 11338 & 1.06 & 2.19 & 75.92 & 327.30 & 2.02 & 8.72 & & 4.50 & 1.55 & 2.45 \\
\hline & & 20.69 & 16853 & 1.01 & 3.43 & 118.17 & 342.66 & 4.12 & 11.94 & & 4.05 & 0.51 & 2.57 \\
\hline & \multirow{3}{*}{ Core } & 6.46 & 5262 & 0.71 & 1.51 & 58.94 & 547.46 & 0.84 & 7.83 & & 2.03 & 1.04 & 4.11 \\
\hline & & 13.54 & 11029 & 0.91 & 2.48 & 96.86 & 429.12 & 2.34 & 10.38 & & 3.31 & 0.94 & 3.22 \\
\hline & & 20.48 & 16682 & 1.11 & 3.08 & 169.08 & 495.32 & 5.04 & 14.75 & & 4.90 & 0.86 & 3.72 \\
\hline & \multirow{3}{*}{ Edwards } & 6.69 & 5449 & 0.70 & 1.59 & 59.69 & 535.61 & 0.93 & 8.33 & & 1.95 & 0.96 & 4.02 \\
\hline & & 13.36 & 10882 & 1.06 & 2.10 & 113.62 & 510.27 & 2.91 & 13.07 & & 4.50 & 1.64 & 3.83 \\
\hline & & 20.57 & 16755 & 1.34 & 2.57 & 191.77 & 559.38 & 5.17 & 15.09 & & 7.13 & 1.90 & 4.20 \\
\hline \multirow{12}{*}{$\begin{array}{c}\text { Dys- } \\
\text { functional } \\
\text { valve }\end{array}$} & \multirow{3}{*}{$\begin{array}{l}\text { Tilting } \\
\text { disc }\end{array}$} & 6.55 & 5335 & 1.47 & 0.75 & 157.35 & 1440.42 & 3.20 & 29.28 & 6.8 & 8.59 & 6.35 & 10.80 \\
\hline & & 13.39 & 10907 & 2.89 & 0.77 & 774.51 & 3471.25 & 11.91 & 53.36 & 30.7 & 33.37 & 24.36 & 26.04 \\
\hline & & 19.83 & 16152 & 4.17 & 0.79 & 2139.28 & 6472.56 & 25.53 & 77.26 & 57.9 & 69.64 & 50.41 & 48.55 \\
\hline & \multirow{3}{*}{ STJM } & 6.56 & 5343 & 1.53 & 0.71 & 149.30 & 1364.59 & 3.01 & 27.50 & 7.6 & 9.39 & 7.03 & 10.24 \\
\hline & & 13.41 & 10923 & 2.96 & 0.76 & 741.85 & 3320.31 & 11.51 & 51.50 & 26.8 & 34.98 & 25.74 & 24.90 \\
\hline & & 20.17 & 16429 & 4.31 & 0.78 & 2069.41 & 6156.25 & 24.56 & 73.07 & 52.6 & 74.47 & 54.22 & 46.18 \\
\hline & \multirow{3}{*}{ Core } & 6.61 & 5384 & 1.02 & 1.08 & 66.09 & 599.96 & 0.97 & 8.77 & 3.7 & 4.17 & 2.64 & 4.50 \\
\hline & & 13.55 & 11037 & 1.52 & 1.49 & 151.85 & 672.39 & 2.75 & 12.18 & 10.3 & 9.19 & 4.75 & 5.04 \\
\hline & & 20.13 & 16397 & 1.72 & 1.95 & 266.61 & 794.82 & 5.59 & 16.66 & 21.1 & 11.84 & 4.74 & 5.96 \\
\hline & \multirow{3}{*}{ Edwards } & 6.96 & 5669 & 1.38 & 0.84 & 114.71 & 989.17 & 2.20 & 19.00 & 1.6 & 7.57 & 5.36 & 7.42 \\
\hline & & 13.89 & 11314 & 2.09 & 1.11 & 298.54 & 1289.37 & 6.48 & 28.00 & 6.8 & 17.40 & 10.89 & 9.67 \\
\hline & & 20.95 & 17065 & 2.49 & 1.40 & 655.85 & 1878.49 & 12.33 & 35.31 & 9.0 & 24.88 & 13.49 & 14.09 \\
\hline
\end{tabular}

Table 2. Summary of linear regression and Bland-Altman analysis 


\begin{tabular}{|c|c|c|c|c|c|c|c|c|}
\hline Method & Slope & $\begin{array}{c}\text { Standard } \\
\text { error of } \\
\text { slope }\end{array}$ & Intercept & $\begin{array}{c}\text { Standard } \\
\text { error of } \\
\text { intercept }\end{array}$ & $\mathbf{R}^{2}$ & P-value & $\begin{array}{c}\text { Mean (Measurement } \\
\text { Prediction) }\end{array}$ & $\begin{array}{c}\text { 1.96SD (Measurement } \\
\text { Prediction) }\end{array}$ \\
\hline $\begin{array}{l}\text { Simplified } \\
\text { Bernoulli }\end{array}$ & 1.17 & 0.11 & 5.28 & 3.15 & 0.946 & $<0.001$ & 8.95 & 13.45 \\
\hline $\begin{array}{l}\text { Extended } \\
\text { Bernoulli }\end{array}$ & 0.87 & 0.06 & 2.66 & 1.80 & 0.969 & $<0.001$ & -0.21 & 8.54 \\
\hline $\begin{array}{l}\text { Turbulence } \\
\text { production }\end{array}$ & 0.75 & 0.02 & 5.44 & 0.70 & 0.993 & $<0.001$ & -0.21 & 10.87 \\
\hline TKE/Q & 0.99 & 0.05 & 21.93 & 1.46 & 0.986 & $<0.001$ & & NA \\
\hline
\end{tabular}


